\title{
The effects of social support and having a partner on sleep quality in dementia
}

\begin{abstract}
Objectives: This study aimed to determine the effects of social support and having a partner on sleep quality in the elderly patients with dementia. Methodology: This research was conducted on 1210 noninstitutionalized elderly Malaysian individuals with dementia. The effects of age, ethnicity, educational level, marital status, sex differences, social support, and having a partner on sleep quality were evaluated in the respondents. The multiple logistic regression analysis was used to predict the risk of sleep disturbances among the participants. Results: Approximately, $41 \%$ of the participants experienced sleep disruption. Further findings showed that ethnicity (odds ratio $[\mathrm{OR}]=0.62)$, social support $(\mathrm{OR}=1.35$ ), marital status $(\mathrm{OR}=2.21)$, educational level $(\mathrm{OR}=0.65)$, and having a partner $(\mathrm{OR}=0.45)$ significantly affected sleep quality $(\mathrm{P}<.05)$. Sex differences and age were unrelated predictors of sleep disturbances $(\mathrm{P}>.05)$. Conclusion: It was concluded that social isolation and being single increased sleep disruption among respondents, but having a partner and ethnic non-Malay decreased the rate of sleep problems.
\end{abstract}

Keyword: Partner; Social support; Sleep quality; Elderly; Dementia 